\title{
Sequence polymorphisms in Pvs48/45 and Pvs47 gametocyte and gamete surface proteins in Plasmodium vivax isolated in Korea
}

\author{
Mi Kyung Woo', Kyeong Ah Kim¹, JuYeon Kim¹, Jun Seo Oh², \\ Eun Taek Han³, Seong Soo A An", Chae Seung Lim¹/+
}

\begin{abstract}
${ }^{1}$ Department of Laboratory Medicine, Korea University Guro Hospital ${ }^{2}$ Laboratory of Cellular Oncology, Brain Korea 21 Graduate School of Medicine, College of Medicine, Korea University, Seoul, Republic of Korea ${ }^{3}$ Department of Parasitology, Kangwon National University College of Medicine, Chuncheon, Republic of Korea ${ }^{4}$ Department of BioNano Technology, Gachon BioNano Research Institute, Gachon University, Seongnam-si, Gyeonggi Do, Republic of Korea
\end{abstract}

Nucleotide sequence analyses of the Pvs48/45 and Pvs47 genes were conducted in 46 malaria patients from the Republic of Korea $(R O K)(n=40)$ and returning travellers from India $(n=3)$ and Indonesia $(n=3)$. The domain structures, which were based on cysteine residue position and secondary protein structure, were similar between Plasmodium vivax (Pvs48/45 and Pvs47) and Plasmodium falciparum (Pfs48/45 and Pfs 47). In comparison to the Sal-1 reference strain (Pvs48/45, PVX_083235 and Pvs47, PVX_083240), Korean isolates revealed seven polymorphisms (E35K, H211N, K250N, D335Y, A376T, I380T and K418R) in Pvs48/45. These isolates could be divided into five haplotypes with the two major types having frequencies of $47.5 \%$ and $20 \%$, respectivelfy. In Pvs 47,10 polymorphisms (F22L, F24L, K27E, D31N, V230I, M233I, E240D, I262T, I273M and A373V) were found and they could be divided into four haplotypes with one major type having a frequency of 75\%. The Pvs $48 / 45$ isolates from India showed a unique amino acid substitution site (K26R). Compared to the Sal-1 and ROK isolates, the Pvs47 isolates from travellers returning from India and Indonesia had amino acid substitutions (S57T and I262K). The current data may contribute to the development of the malaria transmission-blocking vaccine in future clinical trials.

Key words: Plasmodium vivax - Pvs48/45 - Pvs47 - polymorphism - malaria vaccine -

transmission blocking vaccine - Republic of Korea

Malaria is a highly infectious disease and has the highest worldwide mortality rate. According to the 2011 World Malaria Report, the number of suspected malaria cases reached 216 million with 655,000 malaria-related deaths in 2010. The prevalence of Plasmodium vivax infection in 2009 was $71 \%$ in South America and $49 \%$ in both Southern Asia and the Western Pacific. In the Republic of Korea (ROK), P. vivax is the only indigenous strain of malaria (Mendis et al. 2001, WHO 2011).

The life cycle of the malaria parasite is divided into asexual and sexual stages. The malaria parasite can be spread from mosquitoes to humans and can reside and multiply in the liver and red blood cells (RBCs). The asexually transmitted parasites in RBCs can then induce malaria symptoms.

To reduce communal malaria infections, a malaria transmission-blocking vaccine (TBV) has been investigated using surface proteins of male and female gametocytes, zygotes and ookinetes from Plasmodium falciparum (Roeffen et al. 1995, 2001, van Schaijk et al. 2006, Outchkourov et al. 2007, 2008). Pfs $48 / 45$ is expressed on the surface of male and female gametocytes/gametes and is a necessary factor for male fertility (van Dijk et al.

Financial support: Korea University, R \& D Grant (2011)

MKW and KAK contributed equally to this work.

+ Corresponding author: malarim@korea.ac.kr

Received 9 August 2012

Accepted 9 October 2012
2001). The Pfs $48 / 45$ protein can induce antibodies against the surface proteins of gametocytes and the monoclonal antibodies against Pfs $48 / 45$ can block the transmission of the parasite to mosquitoes (Carter et al. 2000, Carter 2001, Stowers \& Carter 2001). With additional supporting results from other investigators, Pfs $48 / 45$ has been accepted as an effective TBV candidate against $P$. falciparum (Roeffen et al. 1995, van Dijk et al. 2001, Drakeley et al. 2006, Pradel 2007). In addition, Pfs47, which is expressed in female gametocytes and gametes, has also demonstrated an ability to induce antibody responses and to serve as a TBV candidate against $P$. falciparum (van Schaijk et al. 2006, Pradel 2007).

Because $P$. vivax is the second most common infectious Plasmodium species, developing a TBV using the surface proteins of gametocytes has been proposed as a possible public health defence mechanism. Pvs48/45 of $P$. vivax, which is a homolog of Pfs $48 / 45$ of $P$. falciparum, is also a TBV candidate (Galinski \& Barnwell 2008). However, TBV research on Pvs $48 / 45$ is in the early stages due to difficulties in producing recombinant Pvs48/45 protein. Pvs47 of $P$. vivax has not yet been investigated as a TBV candidate (Galinski \& Barnwell 2008). Hence, additional studies using the Pvs $48 / 45$ and Pvs47 proteins of $P$. vivax should be performed in the development of a $P$. vivax TBV.

$P$. vivax malaria is an endemic disease in the ROK. The incidence of $P$. vivax decreased sharply with the launch of the WHO malaria control project resulting in an almost complete eradication of $P$. vivax. Unexpectedly, $P$. vivax re-emerged in 1993 and malaria cases climbed 
to 4,141 by 2000 (Yeom et al. 2005). Fortunately, the number of patients with malaria decreased to 841 in 2011. Malaria transmission in the ROK occurs mostly in the regions near the Delimited Militarised Zone (DMZ). Hence, this study was conducted in Kimpo and Pajoo, which are high-risk malaria areas, located in the northwest region of the ROK near the DMZ. Korean $P$. vivax infection presents with mild, but characteristic malaria symptoms. From June-October, the majority of malaria transmission occurs in areas near the DMZ (Yeom et al. 2005). Vector control and chemoprophylaxis have been performed in the ROK near the DMZ, but the malaria outbreaks are not controlled in North Korea. To control the $P$. vivax malaria infection rate on the Korean peninsula, use of the malaria TBV against mosquito hosts in risky areas would be an ideal and long-term way to eliminate malaria in both regions. Therefore, we analysed the polymorphisms in the Pvs48/45 and Pvs47 proteins from the clinical $P$. vivax isolates from the ROK. The results from these Korean isolates may provide valuable baseline data for the development of a TBV and future clinical trials.

\section{SUBJECTS, MATERIALS AND METHODS}

This study was conducted between 2006-2010 at the Korea University Hospital at Guro, ROK. Whole blood samples were collected into ethylenediaminetetraacetic acid tubes from febrile patients for malaria diagnosis. The confirmed samples, which were infected with indigenous Korean $P$. vivax according to Giemsa-stain microscopic analysis, were included in this study. The patients had no history of travel to a foreign country within three years prior to the study. The patients were $22-49$ years old (mean $=37.6$ years) with a male/female ratio of 5.5:1. The following samples were collected: seven in 2006, 16 in 2007, eight in 2008 and nine in 2010. Each patient with malaria received treatments of oral chloroquine $(800 \mathrm{mg}$ of hydroxychloroquine sulphate) followed by $400 \mathrm{mg}$ after $6 \mathrm{~h}, 24 \mathrm{~h}$ and $48 \mathrm{~h}$ for a total of 2,000 mg. After completing chloroquine therapy, oral primaquine was prescribed at $15 \mathrm{mg}$ /day for 14 days. All of the patients responded well to the standard chloroquine therapy. The parasitaemia level was indirectly calculated by assessing the number of parasites per 200 white blood cells (WBCs) in the blood film and using the results of the WBC counts from an automated haematology analyser (Cell-Dyn 4000; Abbott Diagnostics, Santa Clara, CA, USA). The mean \pm standard deviation parasitaemia level was $4,236( \pm 13,210) / \mu \mathrm{L}$ with a range of $46-31,000 / \mu \mathrm{L}$. These patients were residents of or had a history of visiting Kimpo $(\mathrm{n}=26)$ and Pajoo $(\mathrm{n}=14)$. Informed consent was obtained before collection of the blood samples. Six isolates from travellers returning from Indonesia and India were compared against the Korean sequences. The blood samples were stored at $-80^{\circ} \mathrm{C}$. The current protocol was approved by the institutional review board of the Korea University Guro Hospital.

The $P$. vivax DNA was extracted from whole blood using an AquaPure Genomic DNA Kit (BioRad Laboratories, USA) according to the manufacturer's instructions. The Pvs48/45 and Pvs47 P. vivax genes were amplified by polymerase chain reaction (PCR) using the indicated primers. For amplification of the $P v s 48 / 45$ gene, the forward and reverse primers were 5'-CACGCAGATTGATGTCGC-3' (CM000453, 289234-289251) and 5'-CAAACAGGTGCACGCGC-3' (CM000453, 290823-290839), respectively. For amplification of the $P v s 47$ gene, the forward and reverse primers were 5'-CACACCACCGCAAACAGG-3' (CM000453, 286169-286186) and 5'-GTGCACATTCCGCGGTTG-3' (CM000453, 287676-287693), respectively. Primers were used at a final concentration of $0.1 \mu \mathrm{M}$ in $25 \mu \mathrm{L}$ of reaction mixture (10 mM Tris- $\mathrm{HCl}, \mathrm{pH} 8.3 ; 50 \mathrm{mM} \mathrm{KCl}$; $1.5 \mathrm{mM} \mathrm{MgCl}$; $0.2 \mathrm{mM}$ deoxyribonucleotide triphosphate) with $1 \mu \mathrm{L}$ of extracted DNA from whole blood and 2.5 units of ExTaq polymerase (TaKaRa Bio Inc, Japan). The PCR mixtures were cycled 35 times using a TaKaRa PCR Thermal Cycler (TaKaRa Bio Inc, Japan) with the following conditions: each cycle included $1 \mathrm{~min}$ of denaturation at $95^{\circ} \mathrm{C}, 1 \mathrm{~min}$ of annealing at $67^{\circ} \mathrm{C}$ for $P v_{s} 48 / 45$ or $65^{\circ} \mathrm{C}$ for $P v_{s} 47$ and $1.5 \mathrm{~min}$ of extension at $72^{\circ} \mathrm{C}$. Amplified DNA fragments of $P v_{s} 48 / 45$ and $P v_{s} 47$ were size fractionated by electrophoresis using a $1.5 \%$ agarose gel containing $0.5 \mathrm{mg} / \mathrm{mL}$ ethidium bromide. Amplified PCR products were gel purified using the QIAGEN DNA purification kit (QIAGEN, Germany) following the manufacturer's instructions. The sequences were determined using the BigDye Terminator v3.1 Cycle Sequencing Kit and ABI 3730XL automatic DNA sequencer. The sequencing primers for the $P v s 48 / 45$ gene were 5'-CGGGTGCTTTAAAAATGGAA-3' (pvs48R2; CM000453, 290138-290157) and 5'-TGTAAAATCTGCGGACGTGA-3' (pvs48F2; CM000453， 290162290181). The sequencing primers for the $P v_{s} 47$ gene were 5'-GAAGAAAGGGGAGGACCAAG-3' (pvs47F2; PVX 083240, 950-969) and 5'-TTTTCCGTTTTCTGCGTCTTT-3' (pvs47R2; PVX_083240, 13361317). The 46 sequences for the $P v_{s} 48 / 45$ and $P v_{s} 47$ genes were aligned with the reference sequence in addition to the Pvs 48 and $P v_{S} 47$ genes from the Sal-1 strain (PVX 083235 and PVX 083240, respectively) using MultAlin software (Corpet 1988), which is a progressive multiple-sequence alignment algorithm. Corrections were carried out manually. The newly obtained sequences were submitted to the GenBank database (accessions JN582344-JN582353 and JQ435579-JQ435660). A phylogenetic tree was constructed based on the maximumlikelihood method from the MEGA 5.0 package (Tamura et al. 2011) and the reliability of each branch was assessed using 500 bootstrap replications. The rate of nucleotide substitutions in pairwise comparisons between haplotypes was computed by the number of synonymous substitutions per synonymous sites and nonsynonymous substitutions per nonsynonymous sites (Nei \& Gojobori 1986) using the MEGA 5.0 package (Tamura et al. 2011).

To predict each domain in Pvs48/45 and Pvs47, the Pvs48/45 (PVX_083235) and Pvs47 (PVX_083240) gene sequences were aligned with the $P f_{s} 48 / 45$ (XM_001350145) and Pfs47 (XM_001350146) gene sequences of $P$. falciparum, respectively, using the MultAlin computer program and analysing secondary structure using Professor_King's program (aber.ac.uk/ phiwww/ 


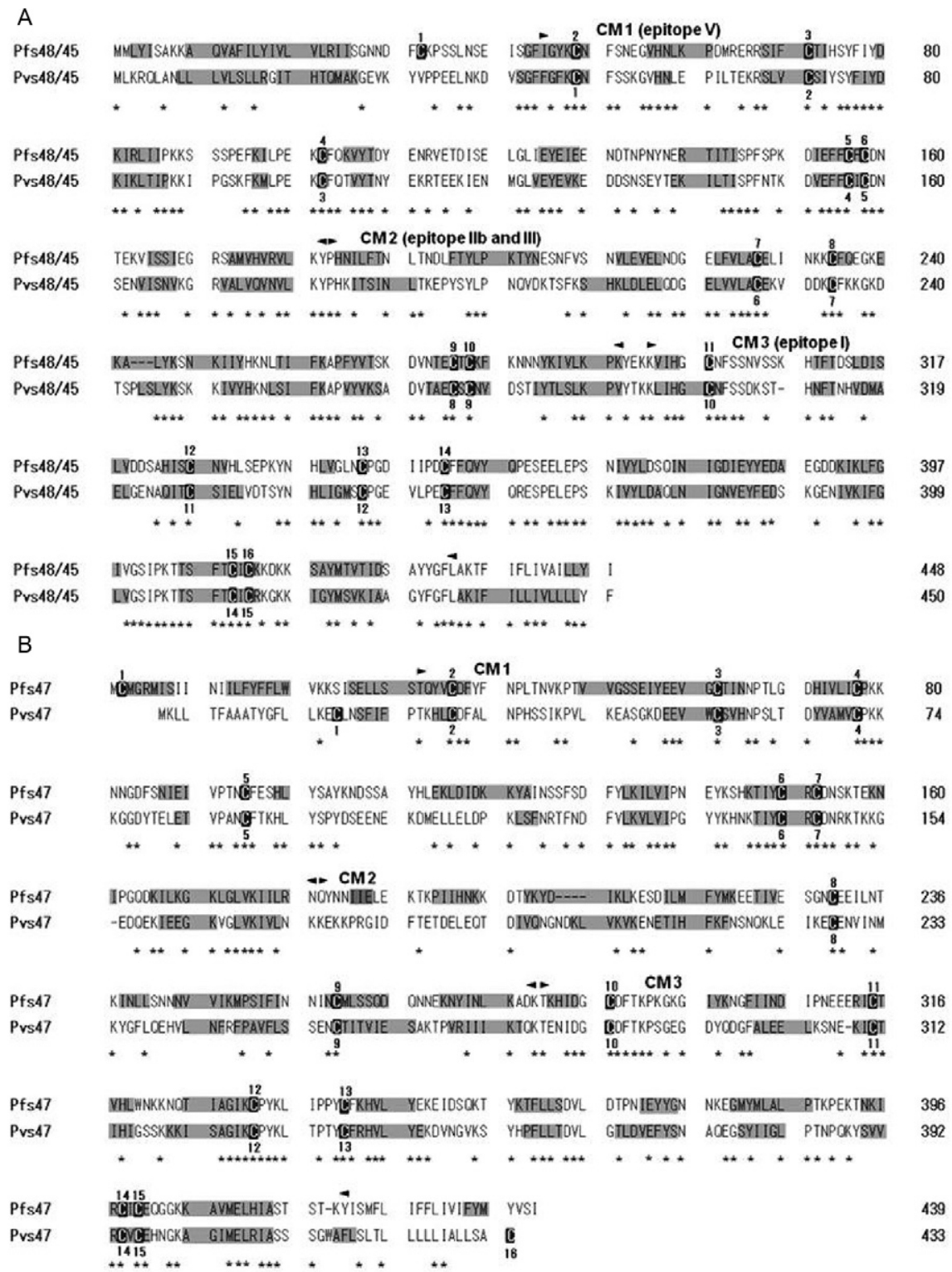

Fig. 1: locations of the cysteine-rich domain (CM)1, CM2 and CM3 domains of Pvs48/45 (A) and Pvs47 (B) in the Plasmodium vivax Sal-1 strain. The locations of the above-mentioned domains were predicted via comparison against the Pfs $48 / 45$ and Pfs 47 sequences of Plasmodium falciparum, respectively, and comparison of the positions of the cysteine residues and $\beta$-sheets (van Dijk et al. 2001, Gerloff et al. 2005). A: three domains in $P f_{s} 48 / 45$ were recognised using different transmission blocking monoclonal antibodies and their targeted epitopes (I, IIb, III and V) were described (Roeffen et al. 2001); B: the Pvs47 epitope was not yet determined; $>$ : range of CM; dark gray: cysteine; light gray: $\beta$-sheet.
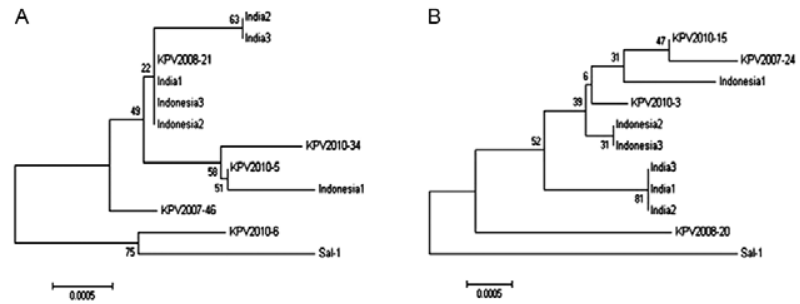

Fig. 2: secondary structure prediction of Pvs48/45 protein. Red colour represented the loop/irregular structures and blue and yellow referred to alpha helical and beta-sheet conformations, respectively. Asterisks indicated the mutations. prof/) (Ouali \& King 2000). Three-dimensional (3D) structure was predicted using the template-based protein structure internet modelling program, RaptorX (Källberg et al. 2012).

\section{RESULTS}

Prediction of cysteine-rich domains (CMs) in $P v s 48 / 45$ and Pvs47 - A 56\% amino acid sequence homology existed between Pvs48/45 (PVX_083235) and Pfs48/45 (PF13_0247) and 42\% homology existed between Pvs47 (PV̄X 083240) and Pfs47 (PF13 0248). The conserved cysteine residues were also found in Pvs48/45 


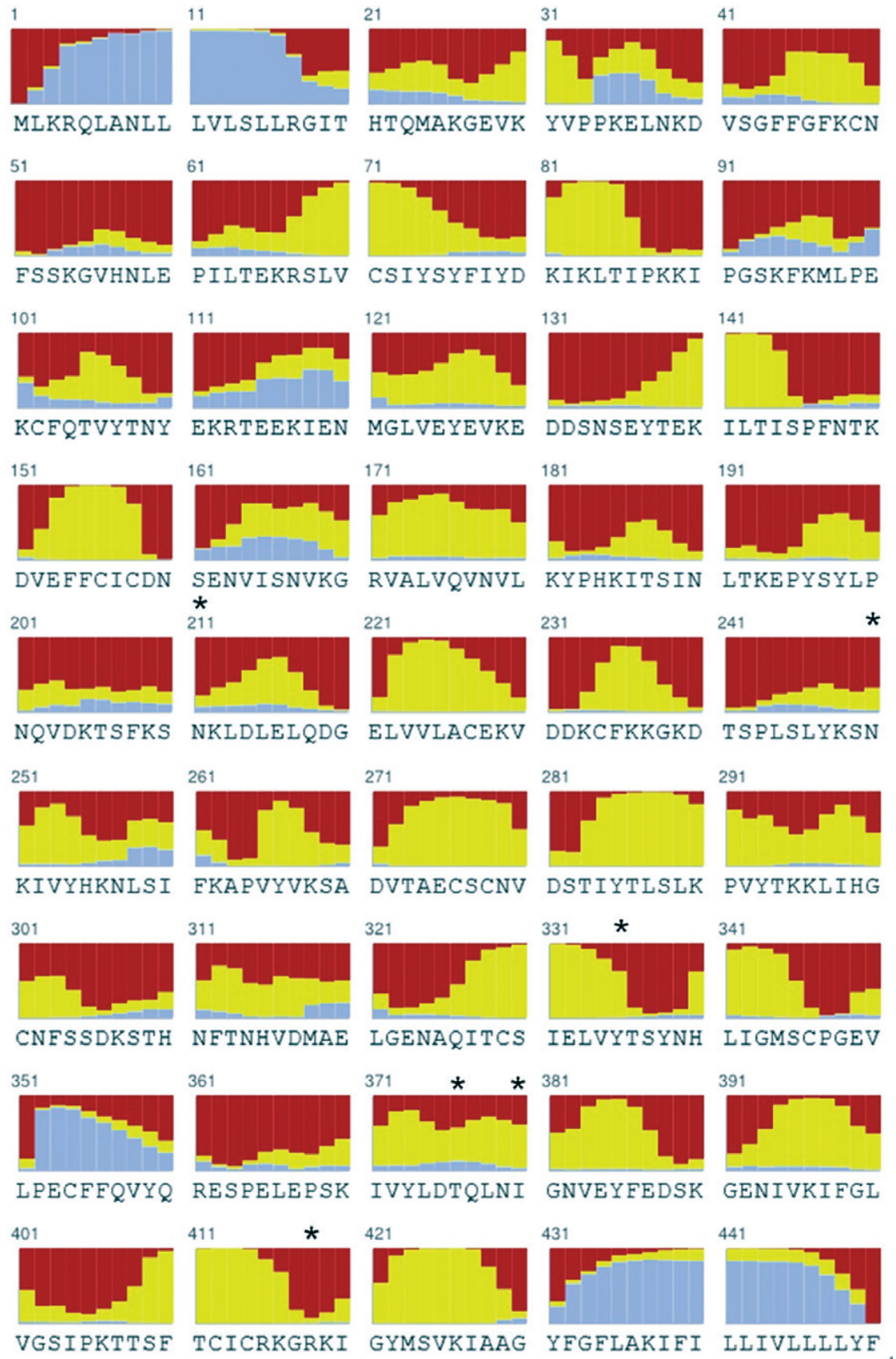

Fig. 3: three-dimensional structure prediction of Pvs48/45 (A-C) and Pvs47 (D-F) proteins. Red coloured atoms represented the mutations in Pvs48/45 (A-C) and blue referred to the mutations in Pvs47 (D-F), respectively.

and Pvs47 (Fig. 1). The predicted domains for comparing the positions of the cysteine residues were found at amino acid positions 45-182 (CM1), 183-292 (CM2) and 296-435 (CM3) in Pvs48/45 and 26-175 (CM1), 176277 (CM2) and 279-417 (CM3) in Pvs47 (van Dijk et al. 2001, Gerloff et al. 2005) (Fig. 1). Pvs48/45 and Pvs47 contained conserved cysteines that formed the disulfide bond in a position similar to that of Pfs $48 / 45$ and Pfs 47 , respectively. Furthermore, in the secondary structures of Pvs48/45, Pvs47, Pfs48/45 and Pfs47, the locations of $\beta$-sheets were similar between $P$. falciparum and $P$. vivax
(Fig. 1). Therefore, the predicted domain and epitope in Pvs48/45 and Pvs47 were similar to those reported for Pfs 48/45 and Pfs47 (Gerloff et al. 2005).

When polymorphisms from Pvs48/45 were used to obtain secondary structure information using RaptorX, a template-based protein structure modelling program (Källberg et al. 2012) positions H211N, K250N and K418R was found to belong to a loop/irregular structure without disorder, which may provide a new epitope for antibody generation (Figs 2, 3A-C). In contrast, positions E35K, D335Y, A376T and I380T were found to belong 


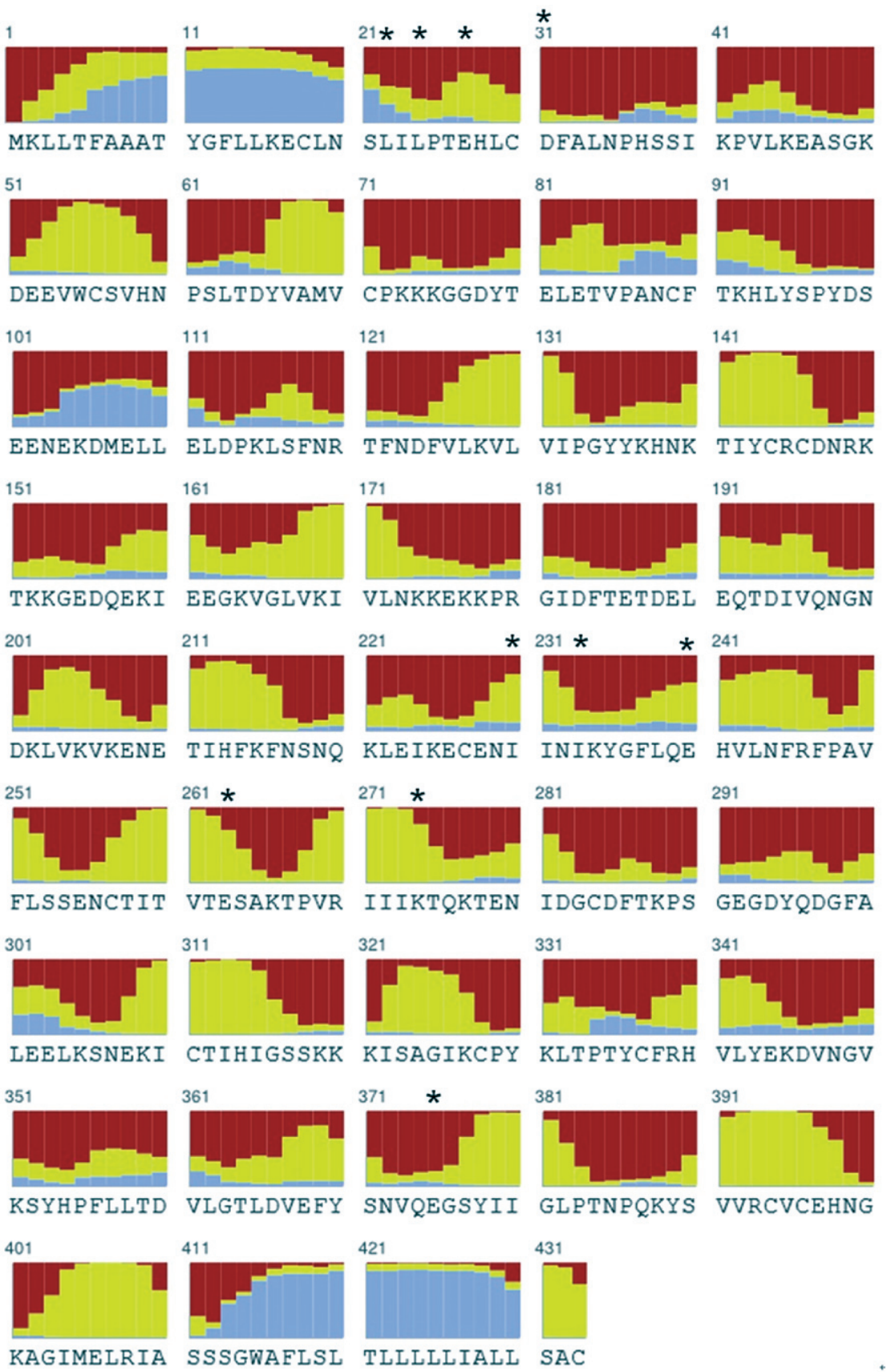

Fig. 4: secondary structure prediction of Pvs47 protein. Red colour represented the loop/irregular structures and blue and yellow referred to alpha helical and beta-sheet conformations, respectively. Asterisks indicated the mutations.

to a beta-sheet region, which caused these residues to be buried in the inner core of the protein, thereby resulting in no accessibility for antibody binding.

For Pvs47, polymorphisms of F22L, F24L, D31N and $\mathrm{A} 373 \mathrm{~V}$ in Figs 3D-F, 4 may fall into the loop regions, whereas mutations V230I, M233I and E240D may participate in forming alpha helix and mutations of $\mathrm{I} 262 \mathrm{~T}$, I273M and $\mathrm{K} 27 \mathrm{E}$ mutations may result in beta-sheet conformations.

Polymorphisms in Pvs48/45 and Pvs47 - From the Pvs48/45 nucleotide and amino acid sequence analyses, one synonymous and eight nonsynonymous substitutions were found in comparison to those in the Sal-1 strain (Table I) in the present study.

In the Korean isolates, seven nonsynonymous substitutions (E35K, H211N, K250N, D335Y, A376T, I380T and K418R) and five haplotypes were found (Table I). The two major types, KPVS48-2010-5 (KNNYTIR) and KPVS48-2010-34 (KNNYTTR), had frequencies of $47.5 \%$ and $20 \%$, respectively (Table I). The two minor types, KPVS48-2010-6 and KPVS48-2008-21, had frequencies of $15 \%$ and $12.5 \%$, respectively (Table I). 
TABLE I

Polymorphisms of Pvs48/45 in Plasmodium vivax isolates from Republic of Korea (ROK), India and Indonesia in comparison with Sal-1 strain

\begin{tabular}{|c|c|c|c|c|c|c|c|c|c|c|}
\hline \multirow{2}{*}{$\begin{array}{l}\text { Position of nucleotides } \\
\text { substitution } \\
\text { (amino acids) }\end{array}$} & \multicolumn{3}{|c|}{ Pre-CM ${ }^{a}$} & \multicolumn{2}{|c|}{ CM2 } & \multicolumn{4}{|c|}{ CM3 } & \multirow[b]{2}{*}{$\begin{array}{c}\text { ROK isolates } \\
n(\%)\end{array}$} \\
\hline & 46 & $\begin{array}{l}77 \\
(26)\end{array}$ & $\begin{array}{l}103 \\
(35)\end{array}$ & $\begin{array}{c}631 \\
(211)\end{array}$ & $\begin{array}{l}750 \\
(250)\end{array}$ & $\begin{array}{l}1003 \\
(335)\end{array}$ & $\begin{array}{l}1126 \\
(376)\end{array}$ & $\begin{array}{l}1139 \\
(380)\end{array}$ & $\begin{array}{l}1253 \\
(418)\end{array}$ & \\
\hline Sal- $1^{b}$ & $\mathrm{C}$ & AAA K & GAG E & CAT H & AAA K & GAT D & GCT A & ATT I & AAG K & - \\
\hline KPVS48-2010-05 (JN582348) & $\mathrm{C}$ & AAA K & AAG K & AAT N & $\mathrm{AAC} \mathrm{N}$ & TAT Y & $\mathrm{ACT} \mathrm{T}$ & ATT I & AGG R & $19(47.5)$ \\
\hline KPVS48-2010-34 (JN582349) & $\mathrm{C}$ & AAA K & AAG K & AAT N & AAC N & TAT Y & ACT T & ACT T & AGG R & $8(20)$ \\
\hline KPVS48-2010-06 (JN582350) & $\mathrm{C}$ & AAA K & GAG E & AAT N & AAC N & GAT D & GCT A & ACT T & AAG K & $6(15)$ \\
\hline KPVS48-2008-21 (JN582352) & $\mathrm{C}$ & AAA K & GAG E & AAT N & $\mathrm{AAC} N$ & TAT Y & ACT T & ATT I & AGG R & $5(12.5)$ \\
\hline KPVS48-2007-46 (JN582353) & $\mathrm{C}$ & AAA K & GAG E & CAT H & $\mathrm{AAC} N$ & TAT Y & ACT T & ATT I & AGG R & $2(5)$ \\
\hline India-1 (JQ435655) & $\mathrm{C}$ & AAA K & GAG E & AAT N & AAC N & TAT Y & ACT T & ATT I & AGG R & - \\
\hline India-2 (JQ435656) & $\mathrm{C}$ & AGA R & GAG E & AAT N & AAC N & TAT Y & ACT T & ATT I & AGG R & - \\
\hline India-3 (JQ435657) & $\mathrm{C}$ & AGA R & GAG E & AAT N & $\mathrm{AACN}$ & TAT Y & ACT T & ATT I & AGG R & - \\
\hline Indonesia-1 (JQ435658) & $\mathrm{T}$ & AAA K & AAG K & AAT N & AAC N & TAT Y & $\mathrm{ACT} \mathrm{T}$ & ATT I & AGG R & - \\
\hline Indonesia-2 (JQ435659) & $\mathrm{C}$ & AAA K & GAG E & AAT N & $\mathrm{AAC} N$ & TAT Y & ACT T & ATT I & AGG R & - \\
\hline Indonesia-3 (JQ435660) & $\mathrm{C}$ & AAA K & GAG E & AAT N & AAC N & TAT Y & ACT T & ATT I & AGG R & - \\
\hline
\end{tabular}

$a$ : this region was located between signal peptide and cysteine-rich domain (CM) $1 ; b$ : reference sequence of Pvs $48 / 45$ of $P$. vivax (Sal-1 strain, PVX_083235). Nucleotides and amino acids polymorphisms were shaded at corresponding positions.

Another haplotype, KPVS48-2007-46, was found in two Korean isolates with a frequency of $5 \%$. In comparison to $S A L-1$ (Table I), six amino acid substitutions at the K26R, H211N, K250N, D335Y, A376T and K418R positions (RNNYTR), were found in the isolates of patients returning from India. Indonesian isolates showed one synonymous substitution at $46 \mathrm{~T}>\mathrm{C}$ and six nonsynonymous substitutions (KNNYTR) at the respective positions. The K26R substitution was found only in the isolates of the patients returning from India and the I380T substitution was found only from Korean isolates.

For the nucleotide and amino acid sequence analysis of Pvs47, two synonymous (78A $>\mathrm{G}$ and $1116 \mathrm{C}>\mathrm{T})$ and 11 nonsynonymous substitutions (F22L, F24L, K27E, D31N, S57T, V230I, M233I, E240D, I262T/K, I273M, A373V) were found in comparison to the Sal-1 strain.

In the Korean $P$. vivax isolates, one synonymous $(78 \mathrm{~A}>\mathrm{G})$ and 10 nonsynonymous substitutions (F22L, F24L，K27E，D31N，V230I，M233I，E240D，I262T, I273M, A373V) were found in comparison to the Sal-1 strain. The Korean isolates revealed four haplotypes. The major haplotype (75\% frequency) was KPVS47-201015 with LLEIITV substitutions (Table II). The second major haplotype (20\% frequency) was KPVS47-2010-3 with LLEIIT substitutions. The two minor haplotypes, KPVS47-2007-24 with LLENIITV substitutions and KPVS47-2008-20 with LLEDTMV substitutions, were found only in a single Korean isolate. In the isolates from the patients returning from India, one synonymous $(78 \mathrm{~A}>\mathrm{G})$ and six nonsynonymous substitutions were found at the following positions: F22L, F24L, K27E,
S57T, M233I, I262T. Among the amino acid substitution sites, the S57T of CM1 was found only in the isolates of the returning patients from India. From the isolates of the patients returning from Indonesia, one synonymous $(78 \mathrm{~A}>\mathrm{G})$ and seven nonsynonymous substitutions were found at F22L, F24L, K27E, V230I, M233I, I262K and A $373 \mathrm{~V}$ positions in comparison with $S A L-1$ (Table II). Compared with ROK and Sal-1 isolates, the Indian and Indonesian isolates had specific amino acid substitutions at S57T and $\mathrm{I} 262 \mathrm{~K}$ in CM1 and CM2, respectively.

The full-length sequences of the 47 isolates of Pvs48/45 and Pvs47 were determined to assess genetic diversity as compared to Sal-1, which is the worldwide $P$. vivax reference sequence. The nucleotide diversity per site was 0.00147 in $P v_{S} 48 / 45$ and 0.00109 in $P v_{S} 47$ with an average number of nucleotide differences of 1.987 and 1.424 , respectively. The synonymous and nonsynonymous nucleotide diversity per site was determined to be 0.00186 and 0.00029 in $P v s 48 / 45$, respectively and 0.00014 and 0.00132 in Pvs47, respectively.

Phylogenetic analysis showed that the Pvs48/45 and Pvs47 haplotypes from the Asian isolates appeared to be scattered without clusters and not influenced by their geographic origins (Fig. 5).

\section{DISCUSSION}

Pvs48/45 and Pvs47 proteins belong to cysteinerich protein families that contain three domains, including A types (CM1 and CM3) and a B type (CM2), with conserved cysteine residue motifs (Gerloff et al. 2005, Pradel 2007). 
TABLE II

Polymorphisms of Pvs47 in Plasmodium vivax isolates from Republic of Korea (ROK), India and Indonesia in comparison with Sal-1 strain

\begin{tabular}{|c|c|c|c|c|c|c|c|c|}
\hline \multirow{2}{*}{$\begin{array}{l}\text { Position of nucleotides } \\
\text { substitution } \\
\text { (amino acids) }\end{array}$} & \multicolumn{2}{|c|}{ Pre- $\mathrm{CM}^{a}$} & \multicolumn{4}{|c|}{ CM1 } & & \\
\hline & $\begin{array}{l}66 \\
(22)\end{array}$ & $\begin{array}{l}70 \\
(24)\end{array}$ & 78 & $\begin{array}{c}79 \\
(27)\end{array}$ & $\begin{array}{c}91 \\
(31)\end{array}$ & $\begin{array}{l}170 \\
(57)\end{array}$ & & \\
\hline Sal- $1^{b}$ & TTC F & TTT F & G & AAG K & GAC D & AGC S & & \\
\hline KPVS47-2010-15 (JN582344) & TTA L & CTT L & A & GAG E & GAC D & AGC S & & \\
\hline KPVS47-2010-03 (JN582345) & TTA L & CTT L & A & GAG E & GAC D & AGC S & & \\
\hline KPVS47-2007-24 (JN582346) & TTA L & CTT L & A & GAG E & $\mathrm{AACN}$ & AGC S & & \\
\hline KPVS47-2008-20 (JN582346) & TTA L & CTT L & A & GAG E & GAC D & AGC S & & \\
\hline India-1 (JQ435615) & TTA L & CTT L & $\mathrm{A}$ & GAG E & GAC D & $\mathrm{ACC} T$ & & \\
\hline India-2 (JQ435616) & TTA L & CTT L & $\mathrm{A}$ & GAG E & GAC D & $\mathrm{ACC} T$ & & \\
\hline India-3 (JQ435617) & TTA L & CTT L & $\mathrm{A}$ & GAG E & GAC D & $\mathrm{ACC} T$ & & \\
\hline Indonesia-1 (JQ435618) & TTA L & CTT L & $\mathrm{A}$ & GAG E & GAC D & AGC S & & \\
\hline Indonesia-2 (JQ435619) & TTA L & CTT L & A & GAG E & GAC D & AGC S & & \\
\hline \multirow[t]{3}{*}{ Indonesia-3 (JQ435620) } & TTA L & CTT L & A & GAG E & GAC D & AGC S & & \\
\hline & \multicolumn{6}{|c|}{$\mathrm{CM} 2$} & CM3 & \\
\hline & $\begin{array}{l}688 \\
(230)\end{array}$ & $\begin{array}{c}699 \\
(233)\end{array}$ & $\begin{array}{l}720 \\
(240)\end{array}$ & $\begin{array}{l}785 \\
(262)\end{array}$ & $\begin{array}{l}819 \\
(273)\end{array}$ & 1116 & $\begin{array}{l}1118 \\
(373)\end{array}$ & $\begin{array}{c}\text { ROK isolates } \\
\text { n (\%) }\end{array}$ \\
\hline Sal- $1^{b}$ & GTT V & ATG M & GAG E & ATA I & ATA I & $\mathrm{T}$ & GCAA & - \\
\hline KPVS47-2010-15 (JN582344) & ATT I & ATT I & GAG E & ACA T & ATA I & $\mathrm{T}$ & GTA V & $30(75)$ \\
\hline KPVS47-2010-03 (JN582345) & ATT I & ATT I & GAG E & ACA T & ATA I & $\mathrm{T}$ & GCAA & $8(20)$ \\
\hline KPVS47-2007-24 (JN582346) & ATT I & ATT I & GAG E & ACA T & ATA I & $\mathrm{T}$ & GTA V & $1(2.5)$ \\
\hline KPVS47-2008-20 (JN582346) & GTT V & ATG M & GAT D & ACA T & ATG M & $\mathrm{T}$ & GTA V & $1(2.5)$ \\
\hline India-1 (JQ435615) & GTT V & ATT I & GAG E & AAA $K$ & ATA I & $\mathrm{T}$ & GCAA & - \\
\hline India-2 (JQ435616) & GTT V & ATT I & GAG E & AAA K & ATA I & $\mathrm{T}$ & GCAA & - \\
\hline India-3 (JQ435617) & GTT V & ATT I & GAGE & AAA K & ATA I & $\mathrm{T}$ & GCA A & - \\
\hline Indonesia-1 (JQ435618) & ATT I & ATT I & GAG E & AAA $K$ & ATA I & $\mathrm{C}$ & GTA V & - \\
\hline Indonesia-2 (JQ435619) & ATT I & ATT I & GAG E & AAA $K$ & ATA I & $\mathrm{T}$ & GCAA & - \\
\hline Indonesia-3 (JQ435620) & ATT I & ATT I & GAG E & AAA K & ATA I & $\mathrm{T}$ & GCAA & - \\
\hline
\end{tabular}

$a$ : this region was located between signal peptide and cysteine-rich domain (CM)1; $b$ : reference sequence of Pvs47 of $P$. vivax (Sal-1 strain, PVX_083240). Nucleotides and amino acids polymorphisms were shaded at corresponding positions.

Four epitopes, including epitope V (CM1), epitope IIb (CM2), epitope III (CM2) and epitope I (CM3), have been constructed in P. falciparum Pfs 48/45 (Gerloffet al. 2005). Based on the results from the membrane-feeding assay, the transmission of parasites is blocked by monoclonal antibodies against epitope I and the effectiveness of blocking transmission by monoclonal antibodies against epitopes IIb, III and V is much weaker (Roeffen et al. 1995, 2001). Other studies have suggested that epitope I (CM3) and epitope III (CM2) may be crucial domains for inducing effective TB antibodies (Outchkourov et al. 2007, 2008). Hence, the baseline data for polymorphisms in CM2 and CM3 would be useful for developing a Pfs $48 / 45$-based TBV. The present results predicted the locations of CM1, CM2 and CM3 in P. vivax Pvs48/45 in comparison to the Sal-1 strain (PVX_083235) (Fig. 1).

Haplotypes with specific substitutions in the CM domains are shown. Nucleotide and amino acid substitutions in Pvs 48/45 from Korean isolates were located at Pre-CM (E35K), CM2 (H211N and K250N) and CM3 (D335Y, 


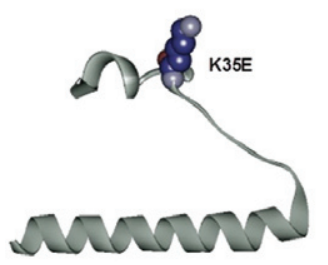

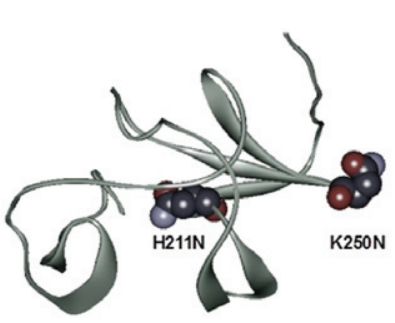

C

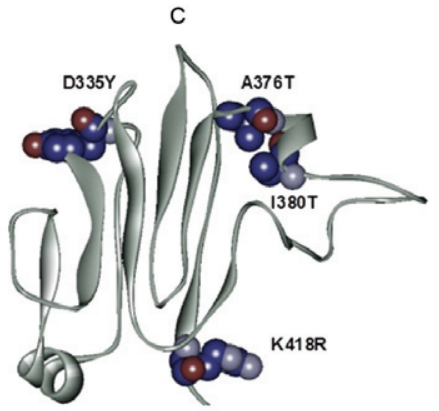

$\mathrm{F}$
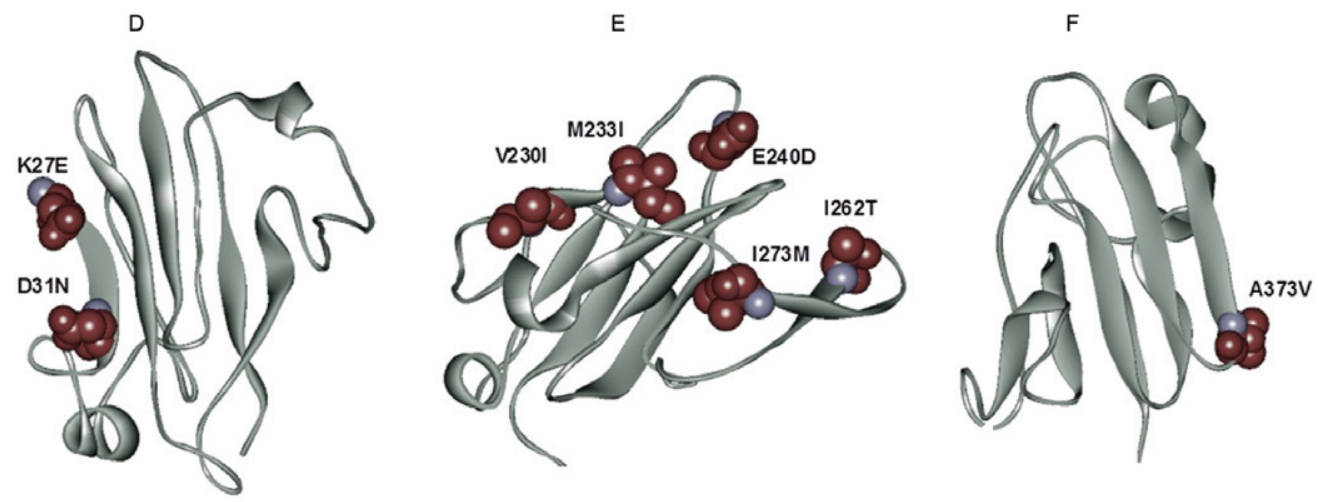

Fig. 5: maximum-likelihood tree of Plasmodium vivax populations from Korea and world isolates inferred from Pvs48/45 (A) and Pvs47 (B) based on pairwise comparison. Values of the bootstrap confidence level are given as per cent branch searches out of 500 replications. The distance corresponding to five changes per 10,000 positions is indicated on the lower left.

A376T, I380T and K418R) as shown in Table I. The substitutions in the isolates from India and Indonesia were also similar to the ROK-type isolates, except for $46 \mathrm{~T}>\mathrm{C}$, K26R and E35K in PreCM, as well as I380T in CM3. In the Pfs48/45 ( $P$. falciparum) polymorphism study by Anthony et al. (2007) five nonsynonymous substitutions were found in CM2 (2 substitutions) and CM3 (3 substitutions). Our results and those from the study by Anthony et al. (2007) revealed no substitution in the CM1 of Pvs48/45 (Table I). As mentioned above, CM2 and CM3 could be highly efficient as a TBV target antigen. Hence, the substitutions in CM2 and CM3 of Pvs48/45 and Pfs48/45 could have occurred as immune protection against the host. Therefore, our polymorphism data and the above epitope studies suggest that CM2 and CM3 of Pvs48/45 could be crucial domain sets for developing an efficient TBV.

Pvs47 also had three domains (CM1, CM2 and CM3), but the results of the epitope study for both Pvs47 and Pfs47 are not yet available. As shown in Table II, two synonymous and 11 nonsynonymous polymorphisms were found. The following substitutions of Pvs47 were found in the Korean isolates: F22L and F24L in Pre-CM, $\mathrm{K} 27 \mathrm{E}$ and D31N in CM1, V230I, M233I, E240D, I262T and I273M in CM2 and A373V in CM3. Among the isolates from India and Indonesia, three substitutions in CM1 (A>G, K27E and S57T) and four substitutions in CM2 (V230I, M233I, I262K and 1666C $>$ T) were found. However, V230I was found only in the isolates from Indonesia. In the study by (Anthony et al. 2007), diverse substitutions were also found in all domains. Therefore, the substitutions in Pvs47 and Pfs47 occurred more often than in Pvs48/45. To test if these substitutions lead to immune protection, an immune response study with recombinant Pvs47 is necessary.

From the 3D protein structure prediction of Pvs48/45, the $\mathrm{H} 211 \mathrm{~N}, \mathrm{~K} 250 \mathrm{~N}$ and $\mathrm{K} 418 \mathrm{R}$ polymorphisms in the loop regions might serve as potential sites for targeted vaccine generation. However, the potential influence of the buried residues on the global protein structure cannot be ruled out, especially if the protein becomes unfolded, thereby exposing the buried residues.

For Pvs47, mutations found in the loop regions, F22L, F24L, D31N, A373V, may directly influence epitope recognition and residues in the alpha helical or beta-sheet conformations may not be directly involved when creating a potent TBV.

The effectiveness of TBV vaccines is dependent on the concentration of the induced antibodies and the quantity of the fertilisation-competent gametocytes. The cysteine residues in the central and C-terminal modules of Pvs48/45 are crucial for proper presentation of the TB epitopes (Outchkourov et al. 2007). However, amino acid substitutions of cysteine residues in Pvs48/45 and Pvs47 were not found in the present study. Therefore, the present results suggested that a TBV based on Pvs48/45 and Pvs47 may induce a TB antibody against antigens with various polymorphisms. Importantly, $P$. vivax isolates in this study were collected between June 2006October 2010. Yearly fluctuations in isolate types were not significant. 
We expect that the polymorphism analyses of Pvs48/45 and Pvs47 of P. vivax from the ROK, India and Indonesia may have significant contributions towards the development of an efficient TBV against Pvs48/45 and Pvs47 proteins in future clinical trials.

\section{REFERENCES}

Anthony TG, Polley SD, Vogler AP, Conway DJ 2007. Evidence of non-neutral polymorphism in Plasmodium falciparum gamete surface protein genes $P f_{s} 47$ and $P f s 48 / 45$. Mol Biochem Parasitol 156: $117-123$.

Carter R 2001. Transmission blocking malaria vaccines. Vaccine 19: 2309-2314.

Carter R, Mendis KN, Miller LH, Molineaux L, Saul A 2000. Malaria transmission-blocking vaccines - how can their development be supported? Nat Med 6: 241-244.

Corpet F 1988. Multiple sequence alignment with hierarchical clustering. Nucleic Acids Res 16: 10881-10890.

Drakeley CJ, Bousema JT, Akim NI, Teelen K, Roeffen W, Lensen AH, Bolmer M, Eling W, Sauerwein RW 2006. Transmissionreducing immunity is inversely related to age in Plasmodium falciparum gametocyte carriers. Parasite Immunol 28: 185-190.

Galinski MR, Barnwell JW 2008. Plasmodium vivax: who cares? Malar $J$ (Suppl. 1): S9.

Gerloff DL, Creasey A, Maslau S, Carter R 2005. Structural models for the protein family characterized by gamete surface protein Pfs230 of Plasmodium falciparum. Proc Natl Acad Sci USA 102: 13598-13603.

Källberg M, Wang H, Wang S, Peng J, Wang Z, Lu H, Xu J 2012. Template-based protein structure modeling using the RaptorX web server. Nat Protoc 7: 1511-1522.

Mendis K, Sina BJ, Marchesini P, Carter R 2001. The neglected burden of Plasmodium vivax malaria. Am J Trop Med Hyg 64: 97-106.

Nei M, Gojobori T 1986. Simple methods for estimating the numbers of synonymous and nonsynonymous nucleotide substitutions. Mol Biol Evol 5: 418-426.

Ouali M, King RD 2000. Cascaded multiple classifiers for secondary structure prediction. Protein Sci 9: 1162-1176.

Outchkourov N, Vermunt A, Jansen J, Kaan A, Roeffen W, Teelen K, Lasonder E, Braks A, van de Vegte-Bolmer M, Qiu LY, Sauer- wein R, Stunnenberg HG 2007. Epitope analysis of the malaria surface antigen pfs $48 / 45$ identifies a subdomain that elicits transmission blocking antibodies. J Biol Chem 282: 17148-17156.

Outchkourov NS, Roeffen W, Kaan A, Jansen J, Luty A, Schuiffel D, van Gemert GJ, van de Vegte-Bolmer M, Sauerwein RW, Stunnenberg HG 2008. Correctly folded Pfs $48 / 45$ protein of Plasmodium falciparum elicits malaria transmission-blocking immunity in mice. Proc Natl Acad Sci USA 105: 4301-4305.

Pradel G 2007. Proteins of the malaria parasite sexual stages: expression, function and potential for transmission blocking strategies. Parasitology 134: 1911-1929.

Roeffen W, Lensen T, Mulder B, Teelen K, Sauerwein R, Van Druten J, Eling W, Meuwissen JH, Beckers PJ 1995. A comparison of transmission-blocking activity with reactivity in a Plasmodium falciparum 48/45-kD molecule-specific competition enzymelinked immunosorbent assay. Am J Trop Med Hyg 52: 60-65.

Roeffen W, Teelen K, van As J, vd Vegte-Bolmer M, Eling W, Sauerwein R 2001. Plasmodium falciparum: production and characterization of rat monoclonal antibodies specific for the sexual-stage Pfs48/45 antigen. Exp Parasitol 97: 45-49.

Stowers A, Carter R 2001. Current developments in malaria transmission-blocking vaccines. Expert Opin Biol Ther 1: 619-628.

Tamura K, Peterson D, Peterson N, Stecher G, Nei M, Kumar S 2011. MEGA5: molecular evolutionary genetics analysis using maximum likelihood, evolutionary distance and maximum parsimony methods. Mol Biol Evol 28: 2731-2739.

van Dijk MR, Janse CJ, Thompson J, Waters AP, Braks JA, Dodemont HJ, Stunnenberg HG, van Gemert GJ, Sauerwein RW, Eling W 2001. A central role for P48/45 in malaria parasite male gamete fertility. Cell 104: 153-164.

van Schaijk BC, van Dijk MR, van de Vegte-Bolmer M, van Gemert GJ, van Dooren MW, Eksi S, Roeffen WF, Janse CJ, Waters AP, Sauerwein RW 2006. Pfs47, paralog of the male fertility factor Pfs 48/45, is a female specific surface protein in Plasmodium falciparum. Mol Biochem Parasitol 149: 216-222.

WHO - World Health Organization 2011. WHO global malaria programme. World malaria report 2011. Available from: who.int/malaria/world_malaria_report_2011/9789241564403_eng.pdf.

Yeom JS, Ryu SH, Oh S, Lee WJ, Kim TS, Kim KH, Kim YA, Ahn SY, Cha JE, Park JW 2005. Status of Plasmodium vivax malaria in the republic of Korea during 2001-2003. Am J Trop Med Hyg 73: 604-608. 